\title{
Multicenter Retrospective Review of Safety and Efficacy of a Novel Minimally Invasive Lumbar Interspinous Fusion Device
}

\author{
Steven M Falowski (D) \\ Vipul Mangal ${ }^{2}$ \\ Jason Pope ${ }^{3}$ \\ Anish Patel ${ }^{4}$ \\ Mark Coleman ${ }^{5}$ \\ Dan Kendall ${ }^{6}$ \\ Richard Brouillette ${ }^{7}$ \\ Michael A Fishman (D) 8 \\ 'Neurosurgical Associates of Lancaster, \\ Lancaster, PA, USA; ${ }^{2}$ National Spine and \\ Pain, National Harbor, MD, USA; ${ }^{3}$ Evolve \\ Restoration, Santa Rosa, CA, USA; \\ ${ }^{4}$ National Spine and Pain, Frederick, MD, \\ USA; ${ }^{5}$ National Spine and Pain, Pikesville, \\ MD, USA; ${ }^{6}$ National Spine and Pain, \\ McLean, VA, USA; ${ }^{7}$ National Spine and \\ Pain, White Plains, MD, USA; ${ }^{8}$ Center for \\ Interventional Pain and Spine, Lancaster, \\ PA, USA
}

\begin{abstract}
Introduction: Several treatment options exist for those with spinal stenosis, as well as degenerative changes. This series evaluates the use of an interspinous fixation (ISF) device as performed by interventional pain physicians.

Methods: This is a retrospective analysis identifying 32 patients with the diagnosis of lumbar degenerative disc disease with secondary diagnosis of lumbar spinal stenosis being treated with ISF with Aurora Spine Zip Interspinous Spacer. Serious adverse events, specifically nerve injury, hematoma, infection, and death, were analyzed quantitatively for reported complications within 90 days from the procedure. In addition, VAS was analyzed for patient reported outcomes.

Results: Adverse event rate was $0 \%$ with no incidences of reoperation, or device removal. Estimated blood loss was recorded as less than $50 \mathrm{cc}$ for all patients. The preoperative pain assessment demonstrated an average pain score of 8.1 and a postoperative pain score of 2.65 equating to a percentage pain reduction of $67 \%$.

Conclusion: This case series demonstrates the success and safety of ISF being performed by interventional pain physicians in an outpatient setting. It is a valuable tool in the treatment of moderate to severe lumbar spinal stenosis and degenerative disc disease that has decreased morbidity and significant efficacy.
\end{abstract}

Keywords: interspinous spacer, indirect decompression, interspinous fusion, interspinous fixation, lumbar degenerative disc disease, spinal stenosis, lumbar stenosis, spinal fusion

\section{Introduction}

Degenerative lumbar spinal stenosis has many etiologies that can include hypertrophied ligamentum flavum, osteophytes, facet joint hypertrophy and degeneration of the disc space. ${ }^{1}$ This is often seen in conjunction with lumbar spinal stenosis, whether secondary to these degenerative factors, or from congenital spinal stenosis. $^{2}$ This cascade of degenerative changes and stenosis is largely the result of aging, as well as the result of the combination of these mentioned factors that contribute.

Several treatment options exist for those with spinal stenosis, as well as degenerative changes. These range from conservative measures to surgical treatments. Conservative measures, or non-surgical treatments generally include physical therapy, medications, and epidural steroid injections. ${ }^{3}$ These are generally reserved for those with mild or moderate symptoms but can also be performed with those who demonstrate further progression. Open laminectomy or decompression of the neural
Correspondence: Steven M Falowski Neurosurgical Associates of Lancaste 160 N Pointe Blvd Suite 200, Lancaster, PA, I760I, USA

Tel + I 7I7-358-0800

Email sfalowski@gmail.com 
structures with or without transpedicular screw fixation has been the treatment most utilized for these patients who fail conservative measures. ${ }^{4}$ However, this may not be appropriate for all patients and has several drawbacks. These may include extended recovery periods from wide muscle dissections and chronic back pain associated with postlaminectomy syndrome. In addition, it may be associated with higher adverse events such as cerebrospinal fluid leak, nerve injury, deep wound infections, misplaced hardware, and hardware failures. ${ }^{5,6}$ It is also well known that posterior lateral fusion is associated with altered spinal dynamics, which can lead to adjacent segment disease and degeneration. ${ }^{7}$ These drawbacks and limitations with more invasive open spinal procedures may limit the patients who can benefit. This is especially true of the cohort of patients who have many medical comorbidities who may be deemed non-surgical candidates, as well as the patient population who may have mild or moderate imaging findings and/or symptoms that are, therefore, considered too early for an open surgical procedure. This leads to the desire to offer minimally invasive options with reduced procedural risks.

Indirect decompression with the use of interspinous process spacers (IPS) has shown positive outcomes and favorable risk profile in the treatment of lumbar spinal stenosis. It limits extension in those patients with stenosis who display symptoms of neurogenic claudication that is relieved in flexion. ${ }^{8}$ However, its use is limited in that these patients are often associated with degenerative changes, spondylolisthesis, and multiple pain generators such as disc degeneration and facet joint hypertrophy, which is not treated by indirect decompression. This has led to the development of minimally invasive devices for interspinous fixation (ISF) which can address both the stenosis and degeneration. This offers an option to patients that have decreased morbidity and potential for equal or comparable efficacy to open surgical decompression with or without fusion. They give the ability to stabilize adjacent spinous processes, decompress neural structures by blocking extension, and minimize overload on adjacent spinal levels.

ISF has been demonstrated to biomechanically give immediate flexion-extension balance and provide effective stabilization for arthrodesis while preserving motion. ${ }^{9,10}$ It is a viable minimally invasive treatment option to those patients not suited for pedicle screw fixation or considered earlier in the treatment paradigm. It has been demonstrated that ISF augmenting anterior interbody placement has several advantages over pedicle screw fixation in terms of skin incisions, invasiveness, muscle dissection, operative times, perioperational outcomes, as well as demonstrates favorable efficacy in reference to visual analog scale (VAS) and Oswestry Disability Index (ODI) in one year follow-ups. ${ }^{11}$ This has also held true in an elderly cohort demonstrating significant improvement in VAS with reliable fusion rates. ${ }^{12}$

Despite these findings it has been considered unclear whether improvement would be obtained without anterior interbody fusion with it being used as a stand-alone device. The use of an ISP without fusion has proved efficacious, safe, and biomechanically maintaining sagittal alignment within a cohort of those with lumbar spinal stenosis. ${ }^{8,13}$ However, it did not directly address the degenerative process commonly associated with those patients, as well as those unable to be treated with an ISP alone. Postacchini et al demonstrated in a prospective study that a stand-alone ISF, with minimally invasive decompression in stenotic patients showing degenerative spondylisthesis, provided fusion in the majority of patients. ${ }^{14}$ More importantly, at the two-year follow-up patients had a highly significant improvement in all outcome measures, indicating a satisfactory clinical result, and none developed instability.

A multicenter randomized controlled trial compared ISF with decompression to decompression alone. ${ }^{15}$ Twoyear follow-up was performed on moderate to severe spinal stenosis. Primary endpoint included a composite of four measures including ODI, secondary surgery or injections, neurological status, and adverse events related to the procedure or device. The primary endpoint was superior for the ISF with decompression group, as well as patients in the decompression alone group being more likely to undergo a secondary intervention or injections. Another multicenter, randomized controlled trial compared ISF with decompression to decompression with pedicle screw fusion. ${ }^{16}$ Moderate to severe lumbar stenosis was included and followed for five years. Composite endpoints included ODI, repeat surgery, further lumbar injections, or adverse events. When comparing both groups the ISF with decompression group had $50.3 \%$ of the patients meeting all four endpoints, while it was at $44 \%$ in the pedicle screw fusion group. The two groups were similar in reoperation rates, as well as improvement in ODI, and VAS. This further demonstrates the utility of ISF.

There are several devices on the market that have been in the surgeon's treatment paradigm for use as ISF devices. 
These devices vary in their application and patient selection. They may coincide with or without a decompression, as well as with or without coinciding anterior spinal fusion. The use of bone graft material is a defining factor in the labeling of ISF. This study is a retrospective analysis, being performed by interventional pain physicians, to evaluate the safety and efficacy of the Aurora Zip standalone ISF device (Aurora Spine, Carlsbad, CA, USA) for the treatment of lumbar spinal stenosis and degenerative disease.

\section{Methods}

WCG Western IRB approval was obtained. All patients provided informed consent, in accordance with the Declaration of Helsinki. All patient data were kept deidentified for confidentiality. The study is exempt under 45 CFR $\S 46.104(d),{ }^{4}$ because the research involves the use of identifiable private information/biospecimens; and information, which may include information about biospecimens, is recorded by the investigator in such a manner that the identity of the human subjects cannot readily by ascertained directly or through identifiers linked to the subjects, the investigator does not contact the subjects, and the investigator will not re-identify subjects.

After a Western IRB exemption was obtained, a retrospective chart review was performed, identifying patients with the diagnosis of lumbar degenerative disc disease with secondary diagnosis of lumbar spinal stenosis and treated with the interspinous interlaminar fusion with Aurora Spine Zip Interspinous Spacer (Aurora Spine). Descriptive statistics were acquired including demographics, procedure characteristics, and preoperative VAS within 15 days from the operative procedure. The incidence of serious adverse events, specifically nerve injury, hematoma, infection, and death was analyzed quantitatively for reported complications within 90 days from the procedure and VAS at 90 days postprocedure.

Patients had to have failed conservative therapy for over six months, which consisted of physical therapy, medications, and injections. All the patients had a MRI within 12 months of the surgery, which identified clear lumbar degenerative disc disease concurrent with at least moderate central canal stenosis defined as $25-50 \%$ canal compromise. All patients received preoperative labs, EKG, and had proper medical clearance to undergo surgery. VAS scores were recorded preoperatively, and all patients had symptoms of low back pain and neurogenic claudication defined as pain improved in flexion and worse with extension or walking. All patients consented for interspinous interlaminar fusion to be done at an ambulatory surgery center.

All patients underwent an interspinous interlaminar fusion with Aurora Spine Zip Interspinous Spacer device. The surgery first consisted of dissection down to the spinous process and removal of interspinous ligament. A ronjeur was used to remove excess bony elements in the interlaminar interspinous space. The industry rasp/sizer was used to size the implant appropriate for the patient and also to promote bony fusion. For all patients, $2 \mathrm{cc}$ of demineralized bone matrix bone graft was utilized inside the barrel of the device and placed in the interspinous space to promote bony fusion. Fluoroscopy was utilized throughout the procedure, with AP and lateral views to confirm final placement.

Figure 1 demonstrates the device in an appropriate position on fluoroscopic imaging.

\section{Results}

The chart review revealed 32 patients that underwent the procedure with follow-up data to 90 days, derived from four interventional pain physicians at two different centers. The 32 cases were over a six-month period. The experience level of the treating physicians varied. All physicians were skilled in typical interventional procedures, however, only two had performed IPS in the past. None of the physicians had experience with ISF. The demographic information, levels treated, and pain scores were identified preoperatively within 15 days of the procedure and within the 90 day postsurgery assessment, including complication assessment of nerve injury, hematoma, infection, and death (Tables 1 and 2). Seven patients underwent two-level interspinous fixation.

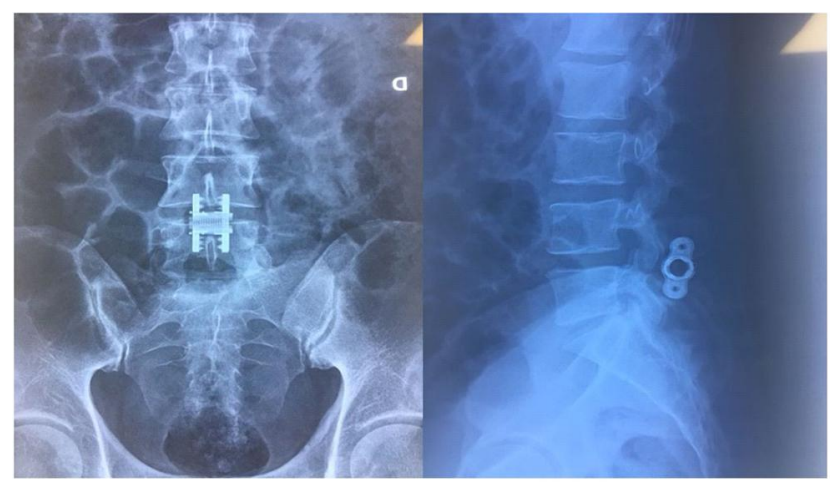

Figure I AP and Lateral Fluoroscopic Imaging of Aurora Zip Interspinous Spacer (Aurora Spine) in the appropriate position. 
Table I Patients Treated with Interspinous Fusion Device Including Outcomes and Complications

\begin{tabular}{|c|c|c|c|c|c|c|}
\hline Age & Gender & Levels & Spinal Stenosis Severity & Preop VAS & 90-Day Postop VAS & $\begin{array}{c}\text { Complication Rate (\%) } \\
\text { Within } 90 \text { Days }^{\mathrm{a}}\end{array}$ \\
\hline 59 & $\mathrm{~F}$ & $\mathrm{~L} 4 / 5$ & Moderate to severe & 8 & 1 & $0 \%$ \\
\hline 67 & M & $\mathrm{L} 3 / 4, \mathrm{~L} 4 / 5$ & Moderate & 7 & 3 & $0 \%$ \\
\hline 78 & M & $\mathrm{L} 3 / 4$ & Moderate & 6 & I & $0 \%$ \\
\hline 89 & $\mathrm{~F}$ & $\mathrm{~L} 4 / 5$ & Moderate & 6 & 5 & $0 \%$ \\
\hline 82 & $\mathrm{~F}$ & $\mathrm{~L} 4 / 5$ & Moderate to severe & 6 & 2 & $0 \%$ \\
\hline 73 & M & $\mathrm{L} 3 / 4, \mathrm{~L} 4 / 5$ & Severe & 8 & 3 & $0 \%$ \\
\hline 61 & $\mathrm{~F}$ & $\mathrm{~L} 4 / 5$ & Moderate & 8 & 4 & $0 \%$ \\
\hline 76 & $\mathrm{~F}$ & $\mathrm{~L} 4 / 5$ & Severe & 10 & 4 & $0 \%$ \\
\hline 70 & $\mathrm{~F}$ & $\mathrm{~L} 4 / 5$ & Moderate & 7 & 3 & $0 \%$ \\
\hline 74 & $\mathrm{~F}$ & $\mathrm{~L} 3 / 4, \mathrm{~L} 4 / 5$ & Moderate & 8 & 3 & $0 \%$ \\
\hline 75 & M & $\mathrm{L} 3 / 4, \mathrm{~L} 4 / 5$ & Severe & 8 & 2 & $0 \%$ \\
\hline 72 & M & $\mathrm{L} 4 / 5$ & Severe & 10 & 4 & $0 \%$ \\
\hline 63 & M & $L 4 / 5$ & Severe & 9 & 4 & $0 \%$ \\
\hline 72 & $\mathrm{~F}$ & $\mathrm{~L} 4 / 5$ & Moderate & 9 & 2 & $0 \%$ \\
\hline 75 & M & $\mathrm{L} 4 / 5$ & Moderate to severe & 8 & 0 & $0 \%$ \\
\hline 76 & M & $\mathrm{L} 3 / 4$ & Moderate & 8 & 4 & $0 \%$ \\
\hline 73 & M & $\mathrm{L} 4 / 5$ & Moderate to severe & 8 & 4 & $0 \%$ \\
\hline 57 & $\mathrm{~F}$ & $\mathrm{~L} 3 / 4$ & Moderate & 10 & 4 & $0 \%$ \\
\hline 68 & M & $\mathrm{L} 3 / 4, \mathrm{~L} 4 / 5$ & Severe & 9 & 3 & $0 \%$ \\
\hline 78 & M & $\mathrm{L} 4 / 5$ & Moderate to severe & 8 & 3 & $0 \%$ \\
\hline 64 & $\mathrm{~F}$ & L3/4, L4/5 & Severe & 7 & 0 & $0 \%$ \\
\hline 7I & M & $\mathrm{L} 4 / 5$ & Severe & 8 & 2 & $0 \%$ \\
\hline 68 & $\mathrm{~F}$ & $\mathrm{~L} 4 / 5$ & Moderate to severe & 10 & 3 & $0 \%$ \\
\hline 63 & $M$ & L3/4, L4/5 & Moderate to severe & 9 & 2 & $0 \%$ \\
\hline 72 & $\mathrm{~F}$ & $\mathrm{~L} 4 / 5$ & Moderate & 7 & 5 & $0 \%$ \\
\hline 72 & M & $\mathrm{L} 3 / 4$ & Moderate to severe & 8 & 0 & $0 \%$ \\
\hline 78 & M & $\mathrm{L} 3 / 4$ & Severe & 8 & 2 & $0 \%$ \\
\hline 82 & $\mathrm{~F}$ & $\mathrm{~L} 4 / 5$ & Moderate & 9 & 4 & $0 \%$ \\
\hline 71 & $\mathrm{~F}$ & $\mathrm{~L} 3 / 4, \mathrm{~L} 4 / 5$ & Moderate to severe & 8 & 2 & $0 \%$ \\
\hline 66 & $M$ & $\mathrm{~L} 4 / 5$ & Moderate & 7 & 3 & $0 \%$ \\
\hline 75 & $\mathrm{~F}$ & $L 4 / 5$ & Moderate to severe & 7 & 2 & $0 \%$ \\
\hline 79 & M & $\mathrm{L} 4 / 5$ & Severe & 9 & 1 & $0 \%$ \\
\hline
\end{tabular}

Note: ${ }^{2}$ Represents occurrence of diagnosed nerve injury, hematoma, infection, death, or allergic reaction to contrast during the decompressive procedure. 
Table 2 Demographics and Outcome Summary

\begin{tabular}{|l|l|}
\hline Number of Participants & 32 \\
\hline Age (mean) & 71.8 \\
\hline Gender & 15 \\
\cline { 1 - 2 } Female & 17 \\
\hline Treated level & \\
\cline { 1 - 1 } Single & 24 \\
Two & 8 \\
\hline Pain assessment & \\
\cline { 1 - 1 } Average preop VAS & 8.1 \\
Average postVAS at 90 days & 2.65 \\
\hline
\end{tabular}

The 32 patients were monitored for complications. No patients had reoperation, or device removal within 90 days. One patient had superficial wound erythema that was treated conservatively with proper wound care that resolved within two weeks. Estimated blood loss was recorded as less than $50 \mathrm{cc}$ for all patients. The preoperative pain assessment demonstrated an average pain score of 8.1 and a postoperative pain score of 2.65 equating to a percentage pain reduction of $67 \%$ (Figure 2).

\section{Discussion}

This consecutive case series demonstrates the experience of four implanting interventional pain physicians who performed minimally invasive ISF in an outpatient ambulatory surgical center (ASC) setting. The experience levels of these physicians varied, but none had previous experience with ISF. This was performed in patients demonstrating low back pain and neurogenic claudication symptoms with imaging findings consistent with moderate to severe degenerative lumbar spinal stenosis at one or two levels. This retrospective review notes a significant reduction in VAS from 8.1 to 2.65 at three-month follow-up, indicating a $67 \%$ reduction in overall pain in this series. Furthermore, the safety demonstrated noted no significant adverse effects at 90 days other than a single incidence of superficial wound erythema that cleared with conservative care. No reoperations or revisions were observed in any case.

Previous studies have shown durable relief from interspinous process spacers in patients with moderate lumbar spinal stenosis followed-up to five years, an outpatient procedure that has been adopted by the interventional pain community. ${ }^{8}$ Studies have demonstrated the superiority of stand-alone ISF in patients suffering from moderate to severe lumbar stenosis compared to decompression alone being performed by surgeons. ${ }^{14,15}$ This case series reproduces the success of

\section{Zip Lumbar Fusion VAS N=32}

9

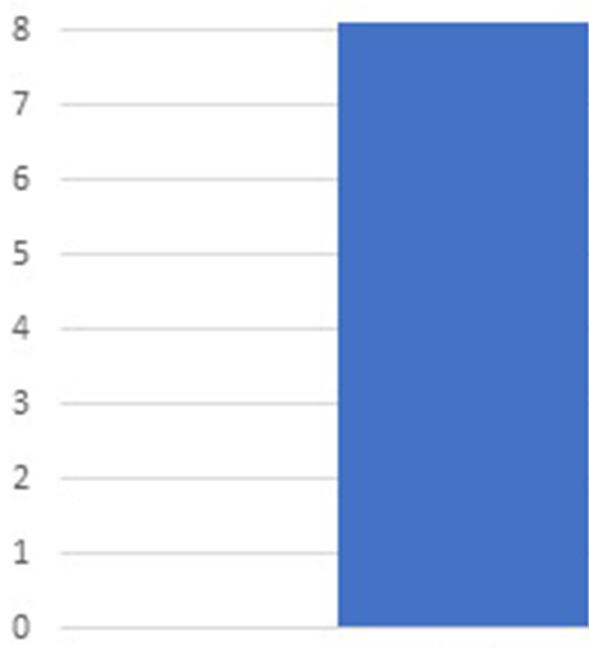

Preop

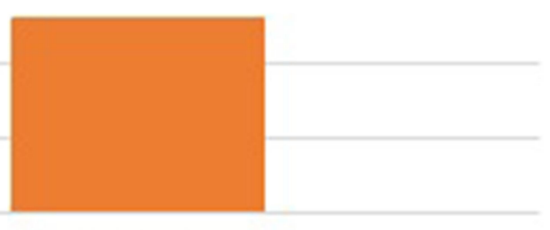

Postop

Preop Dostop

Figure 2 Outcome of VAS Scores for patients at 90 days. 
ISF in a similar patient population being performed by interventional pain physicians in an outpatient setting without prior ISF experience.

The minimally invasive nature of the procedure allowed for successful implantation of the ISF device (Aurora Spine Zip $^{\circledR}$, Carlsbad, CA, USA) in an outpatient ASC, thus avoiding the need for the costs and resources of a hospital setting. In addition, the safety profile included zero complications, revisions, or reoperations at 90 days from four implanting interventional pain physicians. This, in combination with a significant reduction in pain scores demonstrates the utility and reproducibility of this device in this setting.

Limitations of the study include its retrospective nature, lack of functional outcome measures, region-specific pain scores, and detailed analysis of patient demographics including quantitative radiographic analysis, and physical examination.

This promising case series adds another potential tool to the armamentarium of the interventional pain physician in the treatment of moderate to severe lumbar spinal stenosis and degenerative disc disease. This broadens the application to degenerative changes, spondylolisthesis, and multiple pain generators such as disc degeneration and facet joint hypertrophy, which is not treated by indirect decompression alone such as with an interspinous spacer. It is an option to patients that have decreased morbidity and significant efficacy. A prospective, multicenter study is planned to further evaluate the efficacy of this implant in terms of a composite patient success endpoint, including function, pain relief, disability, and adverse events.

\section{Author Contributions}

All authors contributed to data analysis, drafting or revising the article, have agreed on the journal to which the article will be submitted, gave final approval of the version to be published, and agree to be accountable for all aspects of the work.

\section{Funding}

There was no financial support for this document.

\section{Disclosure}

Steven Falowski consults for Abbott, Medtronic, Aurora, Boston Scientific, Vertos and Saluda. Research is performed with Abbott, Medtronic, Biotronik, Saluda, and Vertiflex. Equity positions held in Saluda, Aurora, PainTeq, CornerLoc, SPR Therapeutics, Thermaquil, Stimgenics, SpineThera, Neural Integrative Solutions, and AGR. Vipul
Mangal reports grants from Medtronic, Aurora Spine, Abbott, Boston Scientific, Vertos, SI Fix, and Stimwave, outside the submitted work. Dr Jason Pope consults for Aurora Spine, Vertos, Vertiflex, Flowonix, Abbott, Medtronic, AIS, SPR Therapeutics, Biotronik, Spark, Thermaquil, Saluda, PainTeq, and WISE, outside the submitted work. He is also the co-founder for Celeri Health. Dr Anish Patel reports personal fees from Medtronic, Nevro, Abbott, Flowonix, Camber Spine, Aurora Spine, Spine BioPharma, AIS Healthcare, Stimwave, and Cornerloc, outside the submitted work. Dr Michael A Fishman reports grants, personal fees, and stock options from Aurora Pain Care, personal fees from Biotronik, Abbott, Braeburn, Foundation Fusion Solutions, Institute for Musculoskeletal Education, and Nevro; grants from Medtronic, holds stock options from Celeri Health, SGX International, and Thermaquil, outside the submitted work. The authors report no other conflicts of interest in this work.

\section{References}

1. Zaina F, Tomkins-Lane C, Carragee E, et al. Surgical versus non-surgical treatment for lumbar spinal stenosis. Cochrane Database Syst Rev. 2016;1:CD010264. doi:10.1002/14651858. CD009704.pub2

2. Siebert E, Pruss H, Klingebiel R, Failli V, Einhaupl KM, Schwab JM. Lumbar spinal stenosis: syndrome, diagnostics and treatment. Nat Rev Neurol. 2009;5(7):392-403. doi:10.1038/nrneurol.2009.90

3. Backstrom KM, Whitman JM, Flynn TW. Lumbar spinal stenosis-diagnosis and management of the aging spine. Man Ther. 2011;16(4):308-317. doi:10.1016/j.math.2011.01.010

4. Bridwell KH, Sedgewick TA, O’Brien MF, Lenke LG, Baldus C. The role of fusion and instrumentation in the treatment of degenerative spondylolisthesis with spinal stenosis. $J$ Spinal Disord. 1993;6:461-472. doi:10.1097/00002517-199306060-00001

5. Esses SI, Sachs BL, Dreyzin V. Complications associated with the technique of pedicle screw fixation. A selected survey of ABS members. Spine. 1993;18:2231-2238; discussion 2239. doi:10.1097/ 00007632-199311000-00015

6. Jutte PC, Castelein RM. Complications of pedicle screws in lumbar and lumbosacral fusions in 105 consecutive primary operations. Eur Spine J. 2002;11:594-598. doi:10.1007/s00586-002-0469-8

7. Hilibrand AS, Robbins M. Adjacent segment degeneration and adjacent segment disease: the consequences of spinal fusion? Spine J. 2004;4:190S-194S. doi:10.1016/j.spinee.2004.07.007

8. Nunley PD, Patel VV, Orndorff DG, Lavelle WF, Block JE, Geisler FH. Five-year durability of stand-alone interspinous process decompression for lumbar spinal stenosis. Clin Interv Aging. 2017;12:1409-1417. doi:10.2147/CIA.S143503. PMID: 28919727. PMCID: PMC5593396.

9. Gonzalez-Blohm SA, Doulgeris JJ, Kamran Aghayev WE, Lee AV, Vrionis FD. Biomechanical analysis of an interspinous fusion device as a stand-alone and as supplemental fixation to posterior expandable interbody cages in the Lumbar Spine. J Neurosurg Spine. 2014;20 (2):209-219. doi:10.3171/2013.10.SPINE13612

10. Karahalios DG, Musacchio MJ. Lumbar interspinous devices: fusion and motion sparing. In: Holly L, Anderson P. editors. Essentials of Spinal Stabilization. Cham: Springer; 2017. doi:10.1007/978-3-31959713-3_25. 
11. Kim HJ, Bak KH, Chun HJ, Oh SJ, Kang TH, Yang MS. Posterior interspinous fusion device for one-level fusion in degenerative lumbar spine disease: comparison with pedicle screw fixation - preliminary report of at least one year follow up. $J$ Korean Neurosurg Soc. 2012;52(4):359-364. doi:10.3340/jkns.2012.52.4.359

12. Vokshoor A, Khurana S, Wilson D, Filsinger P. Clinical and radiographic outcomes after spinous process fixation and posterior fusion in an elderly cohort. Surg Technol Int. 2014;25(November):271-276.

13. Falowski S, Sayed D, Deer T, Brescacin D, Liang K. Biomechanics and mechanism of action of indirect lumbar decompression and the evolution of a stand-alone spinous process spacer pain medicine. Pain Med. 2019;20(S2):S14-S22. doi:10.1093/pm/pnz129

14. Postacchini F, Postacchini R, Menchetti PP, Sessa P, Paolino M, Cinotti G. Lumbar interspinous process fixation and fusion with stand-alone interlaminar lumbar instrumented fusion implant in patients with degenerative spondylolisthesis undergoing decompression for spinal stenosis. Asian Spine J. 2016;10(1):27-37. doi:10.4184/asj.2016.10.1.27
15. Schmidt S, Franke J, Rauschmann M, Adelt D, Bonsanto M, Sola S Prospective, randomized, multicenter study with 2-year follow-up to compare the performance of decompression with and without interlaminar stabilization. $J$ Neurosurg Spine. 2018;28(4):406-415. doi:10.3171/2017.11.SPINE17643

16. Musacchio MJ, Lauryssen C, Davis RJ, et al. Evaluation of decompression and interlaminar stabilization compared with decompression and fusion for the treatment of lumbar spinal stenosis: 5-year follow-up of a prospective, randomized, controlled trial. Int J Spine Surg. 2016;10:6. doi:10.14444/3006. PMID: 26913226. PMCID: PMC4752012.

\section{Publish your work in this journal}

The Journal of Pain Research is an international, peer reviewed, open access, online journal that welcomes laboratory and clinical findings in the fields of pain research and the prevention and management of pain. Original research, reviews, symposium reports, hypothesis formation and commentaries are all considered for publication. The manuscript management system is completely online and includes a very quick and fair peer-review system, which is all easy to use. Visit http:// www.dovepress.com/testimonials.php to read real quotes from published authors. 